\title{
Evolutionary patterns of piRNA-generating clusters in human genome
}

O. Dolgova ${ }^{1 *}$, R. Mulet ${ }^{2}$, L. Llobet ${ }^{3}$, S. Casillas ${ }^{2}$, T. Vavouri ${ }^{3}$

${ }^{1}$ Population Genomics Team, Centre Nacional d'Anàlisi Genòmica, Barcelona, Spain

${ }^{2}$ Institut de Biotecnologia i Biomedicina and Department de Genètica i Microbiologia, Universitat Autònoma de Barcelona, Barcelona, Spain

${ }^{3}$ Josep Carreras Leukaemia Research Institute, Barcelona, Spain

*e-mail:olga.dolgova@cnag.crg.eu

Key words: piRNA, human genome, natural selection

Motivation and Aim: PIWI-interacting RNA (piRNA) is the largest class of small non-coding RNA molecules expressed the germline of animals. Their best understood function is genome defense against transposable elements [1]. Most piRNA-generating loci are found in clusters, from which piRNAs are transcribed. Our primary objective was to revisit previously published results, extending them to different populations and chromosome databases, and elucidate which selective forces are mainly acting on piRNA cluster regions in the human genome at the nucleotide level.

Methods and Algorithms: 1000 Genomes Project [2] in its GRCh37 version was chosen for this study as the main dataset, from which piRNA clusters and the nearest intergenic regions, as putatively neutral for all genetic comparisons, were extracted following a newly developed pipeline. Polymorphism, mutation rate and divergence from the chimpanzee genome were calculated for both piRNA clusters and intergenic regions in each of five super-populations, and tests for neutrality and selection were conducted using $\mathrm{R}$ and the PopGenome package.

Results: A strong difference in the levels of polymorphism was found between piRNA clusters and intergenic regions, the latter being more diverse. The values of alpha in the McDonald and Kreitman test were negative in 53-60\% and positive 40-47\% of significant results, suggesting recurrent directional selection fixing new advantageous alleles in some clusters.

Conclusion: Altogether, our results are in accordance with the previous findings [3, 4] that purifying selection is supposed to be the main force driving the evolution of piRNA clusters at the nucleotide level in humans. Nevertheless, directional selection also takes place in a substantial part of piRNA clusters, allowing the occurrence of an evolutionary "arm race" between these functional genomic regions and transposable elements.

\section{References}

1. Iwasaki Y.W., Siomi M.C., Siomi H. (2015) PIWI-Interacting RNA: Its Biogenesis and Functions. Annu Rev Biochem. 84:405-33.

2. 1000 Genomes Project Consortium et al. (2012) An integrated map of genetic variation from 1,092 human genomes. Nature. 471:56-65.

3. Lukic S., Chen K. (2011) Human piRNAs are under selection in Africans and repress transposable elements. Mol Biol Evol. 28:3061-3067.

4. Gould D.W., Lukic S., Chen K.C. (2012) Selective constraint on copy number variation in human piwiinteracting RNA Loci. PloS One. 7(10):e46611. 